\title{
Dynamical Analysis of a Novel 4-D Hyper-Chaotic System With One Non- Hyperbolic Equilibrium Point and Application in Secure Communication
}

Pushali Trikha, Jamia Millia Islamia, India

Lone Seth Jahanzaib, Jamia Millia Islamia, India

\begin{abstract}
In this article, a novel hyper-chaotic system has been introduced and its dynamical properties (i.e., phase plots, time series, lyapunov exponents, bifurcation diagrams, equilibrium points, Poincare sections, etc.) have been studied. Also, the novel chaotic systems have been synchronized using novel synchronization technique multi-switching compound difference synchronization and its application have been shown in the field of secure communication. Numerical simulations have been undertaken to validate the efficacy of the synchronization in secure communication.
\end{abstract}

\section{KEYWORDS}

Dynamical Analysis, Multi-Switching Compound Difference Synchronization, Non-Hyperbolic Equilibrium Point, Novel Hyper-Chaotic System, Secure Communication

\section{INTRODUCTION}

The study of chaotic systems to unveil their complex and rich dynamics has gained strength ever since the Lorenz system (Luo, 2009) (Mahmoud \& Mahmoud, 2010)(Wu, Zhang \& Zhong, 2019) was discovered in 1960. The scientific community worldwide got interested in taming chaos (total randomness) either by synchronizing chaotic systems or controlling chaos (Khan \& Bhat, 2016) (Singh, Yadav \& Das,2016). Synchronization (1990) (Pham, Jafari, Wang \& Ma, 2016)(Mahmoud $\&$ Mahmoud, 2012) involves the process of locking the dynamics of one system into the other. Many synchronization techniques have been developed such as complete synchronization, antisynchronization (Jimenez-Triana, Chen \& Gauthier, 2015) (Karavaev, Kulminskiy, Ponomarenko \& Prokhorov, 2015), hybrid synchronization (Khan, Khattar \& Prajapati, 2017) (Khan \& Bhat, 2017), projective synchronization, function projective synchronization, modified function projective synchronization, phase synchronization (Jafari, Pham \& Kapitaniak, 2016), lag synchronization (Jafari, Sprott \& Molaie, 2016)(Guo, 2011), combination synchronization, combination-combination synchronization, compound synchronization etc (Khan, Khattar \& Prajapati, 2017)(Khan \& Tyagi, 
2018)(Khan \& Singh, 2017) (Kousaka, Ueta \& Kawakami, 1999)(Li, Hu, Sprott \& Wang, 2015) using various control techniques such as Active Control, Adaptive Control (Park, 2005)(Torrieri,1985) (Vaidyanathan,2014), Sliding Mode Control, Tracking Control, Optimal Control (Skardal, SevillaEscoboza, Vera-Aviia \& Buldu, 2017) etc. (Othman, Noorani \& Al-sawalha,2017)(Ghabi, Rhif \& Vaidyanathan, 2018) (Bouzaida \& Sakly, 2018) (Balochian \& Rajaee, 2018)

\subsection{Prior Work}

Nowadays new chaotic systems whose dynamics relate with that of some dynamical system present in nature are being proposed and analyzed by researchers to have a better understanding of such systems (Qin \& Chen, 2007) (Li, Sprott \& Thio, 2015)(Lu, Chen, Cheng \& Celikovsky, 2002). Many higher dimensional systems have been introduced and studied extensively. Various types of systems (chaotic and hyper chaotic) with different phase space (double scroll, multi-scroll etc.) have been designed by adding the trigonometric functions, switching function, piecewise linear function to the existing systems. The purpose of designing these type of system is to amend the complications of the original system. Also systems are classified on the basis of their equilibrium points as they play an important role in studying chaotic systems. Some systems are reported of having finite number of equilibrium points, some with infinite number of equilibrium points and some with no equilibrium point at all. Systems not related to modeling of natural phenomenon are also being proposed by authors because of their simple structure and unique properties. Also such systems and suitable application in the field of secure communication (Khan \& Bhat, 2017) (Chang, Li, Wang \& Zeng, 2018) (Feki, 2003) (Zabihi, Kiranyaz, Rad, Katsaggelos, Gabbouj \& Ince, 2016), image encryption (Wolf, Swift, Swinney \& Vastano, 1985) (Wu, Noonan, Yang \& Jin, 2012)(Vaidyanathan, Volos, Kyprianidis, Stouboulos \& Pham) as these are difficult to be hacked and are highly sensitive to initial conditions and parameter values.

Motivated by the above discussions, a new hyper-chaotic system has been constructed here. The contribution and novelty of this article are:

1. A new 4-D hyper-chaotic system has been constructed. The beauty of this system lies in its simple structure and complex dynamics.

2. The existence, uniqueness and boundedness of the solution of the new system has been discussed.

3. Continuous dependence on initial condition has been discussed which would help us to find a region where system is not chaotic.

4. The standard dynamical analysis has been discussed like equilibrium points and their stability, bifurcation diagrams, phase portraits, lyapunov exponents etc.

5. A novel synchronization technique "Multi-switching Compound Difference Synchronization" has been adopted. The numerical simulations verify the theoretical analysis provided.

6. The application of synchronization technique in secure communication has been discussed and verified by taking an example.

The rest of the article is arranged as:

Section 2: constructs the novel 4-D hyper-chaotic system. Section 3: comprises of the dynamic analysis of the newly constructed system. To elaborate 3.1 discusses the boundedness of solutions. 3.2 its symmetry. 3.3 discusses the dissipative property of the system (Patra, Hoover, Hoover \& Sprott, 2016). 3.4 shows the existence and uniqueness of the system's solution. 3.5 finds a region where the system would not be chaotic by using its continuous dependence on initial conditions. 3.6 examines for equilibrium points of the system and their stability (Pecora \& Carroll, 1990) (Jiang, Liu \& Luo, 2014) (Jiang, Zhang \& Li, 2018) (Wang, Teng \& Qin, 2012). 3.7 determines the Lyapunov Exponents (Wei, 2011) (Geist, Parlitz \& Lauterborn, 1990) and the Kaplan-Yorke dimension (Evans, Cohen, Searles \& Bonetto, 2000) of the system. 3.8 displays the Poincare section (Yu, 1999) and bifurcation analysis. (Broucke, 1987) (Li \& Sprott, 2014). Section 4: synchronizes the new chaotic 
system in multi-switching compound difference manner. Section 5: shows the utilization of the novel chaotic system in the field of secure communication (Tabor \& Weiss, 1981) using the above designed synchronization scheme (Seyedzadeh \& Mirzakuchaki, 2012). Secrion 6: concludes the article.

\section{CONSTRUCTION OF NOVEL CHAOTIC SYSTEM}

We here introduce a novel hyper-chaotic system given by:

$$
\begin{aligned}
& \dot{x}_{1}=x_{2} \\
& \dot{x}_{2}=a x_{3} \\
& \dot{x}_{3}=x_{4} \\
& \dot{x}_{4}=-b x_{3}-x_{4}+g x_{2}\left(1-c x_{1}^{2}+d x_{1}^{4}-e x_{1}^{6}\right)
\end{aligned}
$$

where $x=\left(x_{1}, x_{2}, x_{3}, x_{4}\right)^{T} \in R^{4}$ are state variables and $\mathrm{a}, \mathrm{b}, \mathrm{c}, \mathrm{d}, \mathrm{e}, \mathrm{g} \hat{\mathrm{I}} R$ are parameters.

For parameter values $a=5, b=24, c=-0.05, d=2.55, e=1.7, g=2.001$ and initial conditions $\left(x_{1}(0) ; x_{2}(0) ; x_{3}(0) ; x_{4}(0)\right)=(-0: 1 ; 0: 2 ;-0: 1 ; 0: 1)$ the corresponding time series and phase portraits of the given system are displayed in Figure 1 and Figure 2 respectively.

\section{DYNAMICAL ANALYSIS OF THE NEWLY CONSTRUCTED HYPER-CHAOTIC SYSTEM}

Here we analyze some of the dynamical properties of the new system viz. boundedness of solutions, symmetry, dissipativity, existence and uniqueness of solution, continuous dependence on initial conditions, equilibrium points, lyapunov exponents, Kaplan-Yorke dimension, bifurcation diagram etc. of the new system.

\subsection{Boundedness of Solutions}

The solutions $x_{i}(t), i=1,2,3,4$ of system (1) are bounded.

The solution $\mathrm{x}_{\mathrm{i}}(t), i=1,2,3,4$ of the system (1) oscillates about the $X$-axis but remain bounded throughout.

\subsection{Symmetry}

The new 4-D hyper-chaotic system remains invariant under the co-ordinate transformation $x_{1}{ }^{\circledR} x_{1}, x_{2}$ ${ }^{\circledR}-x_{2}, x_{3}{ }^{\circledR}-x_{3}$ and $x_{4}{ }^{\circledR}-x_{4}$. Thus, the system shows symmetric behavior about the $x_{1}$ axis.

\subsection{Dissipativity}

System (1) can be expressed as:

$$
\dot{X}=S(X)
$$

where $X=\left(x_{1} ; x_{2} ; x_{3} ; x_{4}\right)^{\mathrm{T}}$ 
Figure 1. a-d Time series plot of state variables $x_{1} ; x_{2} ; x_{3} ; x_{4}$ of system (1) respectively

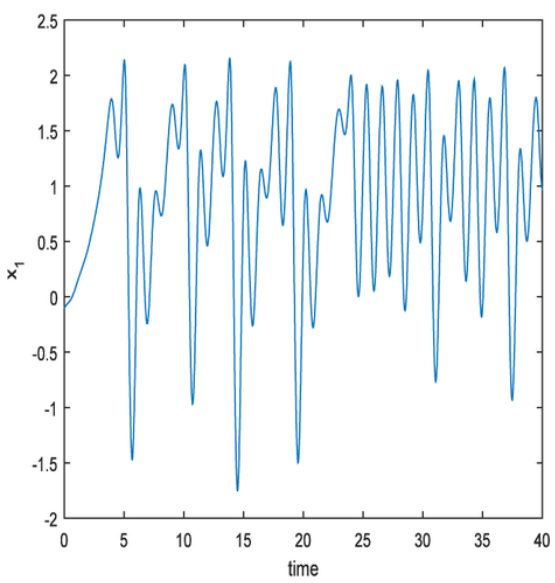

(a)

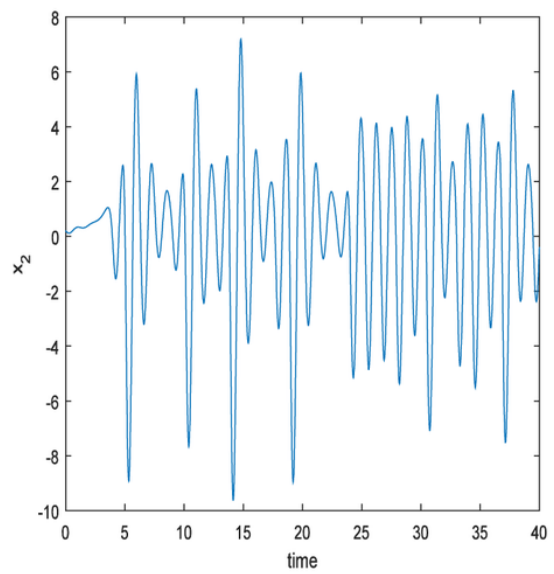

(b)

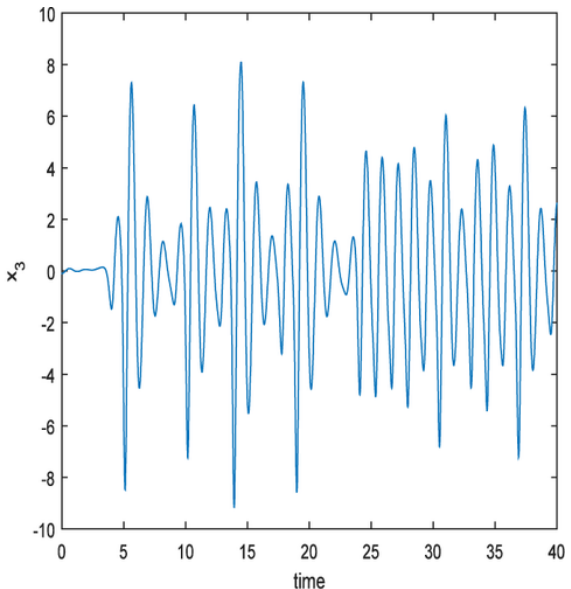

(c)

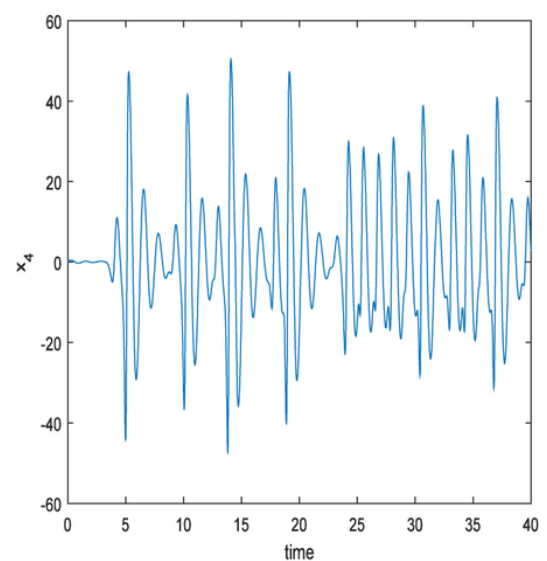

(d)

i.e. $\left[\begin{array}{c}\dot{x}_{1} \\ \dot{x}_{2} \\ \dot{x}_{3} \\ \dot{x}_{4}\end{array}\right]=\left[\begin{array}{l}S_{1}\left(x_{1}, x_{2}, x_{3}, x_{4}\right) \\ S_{2}\left(x_{1}, x_{2}, x_{3}, x_{4}\right) \\ S_{3}\left(x_{1}, x_{2}, x_{3}, x_{4}\right) \\ S_{4}\left(x_{1}, x_{2}, x_{3}, x_{4}\right)\end{array}\right]$

where:

$S_{1}\left(x_{1}, x_{2}, x_{3}, x_{4}\right)=x_{2}$

$S_{2}\left(x_{1}, x_{2}, x_{3}, x_{4}\right)=a x_{3}$

$S_{3}\left(x_{1}, x_{2}, x_{3}, x_{4}\right)=x_{4}$

$S_{4}\left(x_{1}, x_{2}, x_{3}, x_{4}\right)=-b x_{3}-x_{4}+g x_{2}\left(1-c x_{1}^{2}+d x_{1}^{4}-e x_{1}^{6}\right)$ 
Figure 2. Phase Portraits of system (1) in (a) $x_{2}-x_{4}-x_{3}$ plane (b) $x_{2}-x_{4}-x_{1}$ plane (c) $x_{1}-x_{2}-x_{3}$ plane (d) $x_{4}-x_{1}-x_{3}$ plane

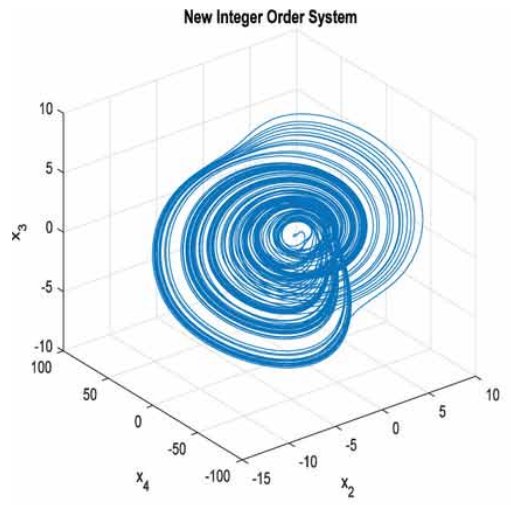

(a)

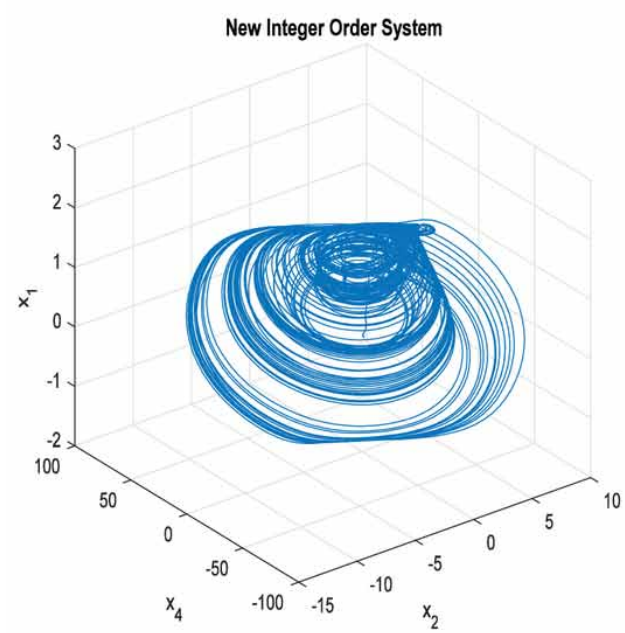

(b)

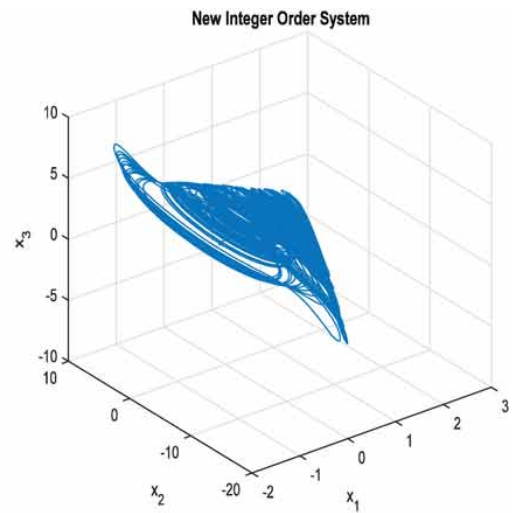

(c)

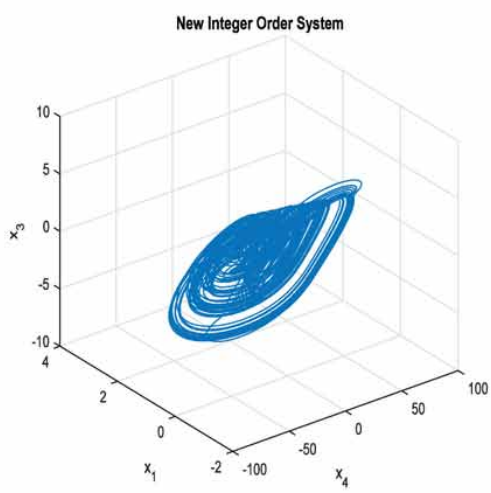

(d)

We consider a region $\Omega$ in $\mathrm{R}^{4}$ with a uniform boundary such that $\omega(t) \in \Psi(t)$, where $\Psi(t)$ is the flow of $S$.

Denoting the volume by $\mathbf{V}(t)$ and then using Liouville's Theorem, we get:

$$
V(t)=\int_{\omega(t)}(\nabla S) d x_{1} d x_{2} d x_{3} d x_{4}
$$

We find the divergence of vector field $S$ by:

$$
\begin{aligned}
& \nabla S=\frac{\partial S_{1}}{\partial x_{1}}+\frac{\partial S_{2}}{\partial x_{2}}+\frac{\partial S_{3}}{\partial x_{3}}+\frac{\partial S_{4}}{\partial x_{4}} \\
& =0+0+0+(-1)
\end{aligned}
$$


i.e. $\nabla S<0$, implying that the system under consideration is dissipative.

Clearly the described system is dissipative for any choice of parameter values a, b, c, d, e, g î $R$. From (2), we have:

$$
\begin{aligned}
& \dot{V}(t)=\int_{\Omega(t)}(-1) d x_{1} d x_{2} d x_{3} d x_{4} \\
& =-V(t)
\end{aligned}
$$

Integrating (3), we get:

$V(t)=\exp (-t) V_{0}$

where $V(0)=\mathrm{V}_{0}$.

Now as $t \circledR ¥ ; V(t) \circledR 0$, i.e. each region containing the trajectory of the system (1) converges to zero implying that with time all the trajectories of the system (1) converge to an attractor.

\subsection{Existence and Uniqueness of the Solution}

The new 4-D hyper-chaotic system can be described as:

$\dot{X}(t)=\Phi(X(t))$

where $t \hat{\mathrm{I}}(0 ; T]$ and its initial values are given by $X(0)=X_{o}$.

Here:

$$
X=\left[\begin{array}{l}
x_{1} \\
x_{2} \\
x_{3} \\
x_{41}
\end{array}\right], X_{o}=\left[\begin{array}{c}
x_{1 o} \\
x_{2 o} \\
x_{3 o} \\
x_{4 o}
\end{array}\right], \Phi(X(t))=\left[\begin{array}{c}
x_{2} \\
a x_{3} \\
x_{4} \\
-b x_{3}-x_{4}+g x_{2}\left(1-c x_{1}^{2}+d x_{1}^{4}-e x_{1}^{o}\right)
\end{array}\right]
$$

We now examine the solution of the system in the region $\omega \times I$, where $I=(0, \mathrm{~T}]$ and $\omega=\left(x_{i}\right): \max \left|x_{1}\right| \leq R, R>0$ for $i=1,2,3,4$. Parameter $\mathrm{R}$ lays a boundary for considering the existence and uniqueness of solution in the required phase space region.

The I.V.P. $X(t)=\Phi(X(t)), X_{O}=X(0)$ is equivalent to:

$$
X(t)=X_{O}+\int_{0}^{t} \Phi(X(s)) d s
$$

Denote: 
$X_{O}+\int_{0}^{t} \Phi(X(s)) d s$ by $H(X), X_{1}=\left[\begin{array}{l}x_{11} \\ x_{12} \\ x_{13} \\ x_{14}\end{array}\right], \quad X_{2}=\left[\begin{array}{c}x_{21} \\ x_{22} \\ x_{23} \\ x_{24}\end{array}\right]$

we get:

$H\left(X_{1}\right)-H\left(X_{2}\right)=\int_{0}^{t}\left(\phi\left(X_{1}(s)-\phi\left(X_{2}(s)\right)\right) d s\right.$

$\mathbf{p}\left|H\left(X_{1}\right)-H\left(X_{2}\right)\right|=\mid \int_{0}^{t}\left(\phi\left(X_{1}(s)-\phi\left(X_{2}(s)\right)\right) d s \mid\right.$

For $h(t) \in C(0, T]$, we define the norm $\|h\|=\operatorname{Sup}_{t \in(0, T)}|h(t)|$.

For the matrix $Q=\left[q_{i j}(t)\right]$ with elements as continuous functions we define the norm $\|Q\|=\sum_{i, j} \operatorname{Sup}_{t \in(0, T]}\left|g_{i, j}(t)\right|:$

$\Rightarrow\left\|H\left(X_{1}\right)-H\left(X_{2}\right)\right\| \leq T \max \left(c g\left|R^{2}+\right| d g\left|R^{4}+\right| e g\left|R^{6}, 1+\right| g|+| c g\left|R^{2}+\right| d g\left|R^{4}+\right| e g \mid R^{6}\right.$,

$|a|+|b|, 2)\left\|X_{1}-X_{2}\right\| \leq R_{1}\left\|X_{1}-X_{2}\right\|$

where:

$R_{1}=T \max \left(|c g| R^{2}+|d g| R^{4}+|e g| R^{6}, 1+|g|+|c g| R^{2}+|d g| R^{4}+|e g| R^{6},|a|+|b|, 2\right)$

Thus $X=H(X)$ is a contraction mapping for sufficient condition $0<R_{1}<1$.

\subsection{Continuous Dependence on Initial Conditions}

We consider two initial conditions $\mathrm{X}_{01}$ and $\mathrm{X}_{02}$ for the system $\dot{X}(t)=\Phi(X(t))$ such that:

$\left\|X_{01}-X_{02}\right\| \leq \delta$

For Condition (4), we consider:

$$
\begin{aligned}
& X_{1}=X_{01}+\int_{0}^{t} \Phi\left(X_{1}(s) d s\right) \\
& X_{2}=X_{02}+\int_{0}^{t} \Phi\left(X_{2}(s) d s\right)
\end{aligned}
$$

We get the following: 
$\left(1-R_{1}\right)\left\|X_{1}-X_{2}\right\| \leq\left\|X_{01}-X_{02}\right\|$

where $0<\mathrm{R}_{1}<1$.

Let $\varepsilon=\frac{\delta}{\left(1-R_{1}\right)}$, then:

$\left\|X_{1}-X_{2}\right\| \leq \varepsilon$

Theorem: The solution of the new system (1) which satisfies (4) would show continuous dependence on initial conditions if $\forall \varepsilon>0 \exists \delta(t)=\left(1-R_{1}\right) \varepsilon>0$ such that $\left\|X_{01}-X_{02}\right\| \leq \delta$, thereby implying $\left\|X_{1}-X_{2}\right\| \leq \varepsilon$.

The above theorem helps in finding a range of parameter values of the system and time $\mathrm{T}$ where chaos does not exist in System (1).

\subsection{Equilibrium Points and Their Stability}

To find the equilibrium points of the system, we equate $S_{1}\left(x_{1}, x_{2}, x_{3}, x_{4}\right), S_{2}\left(x_{1}, x_{2}, x_{3}, x_{4}\right)$, $S_{3}\left(x_{1}, x_{2}, x_{3}, x_{4}\right)$ and $S_{4}\left(x_{1}, x_{2}, x_{3}, x_{4}\right)$, to zero, i.e.:

$$
\begin{aligned}
& x_{2}=0 \\
& a x_{3}=0 \\
& x_{4}=0 \\
& -b x_{3}-x_{4}+g x_{2}\left(1-c x_{1}^{2}+d x_{1}^{4}-e x_{1}^{6}\right)=0
\end{aligned}
$$

For $a=5, b=24, c=-0.05, d=2.55, e=1.7, g=2.001$ we obtain the only equilibrium point $E=(0.885493,0,0,0)$.

Theorem: The equilibrium point $\mathrm{E}$ for the chosen parameter values is unstable, non-hyperbolic equilibrium point.

Proof: The Jacobian matrix of the system is given by:

$$
\begin{gathered}
J=\left[\begin{array}{cccc}
0 & 1 & 0 & 0 \\
0 & 0 & a & 0 \\
0 & 0 & 0 & 1 \\
-2 c g x_{1} x_{2}+4 d g x_{2} x_{1}^{3}-6 e g x_{2} x_{1}^{5} & g\left(1-c x_{1}^{2}+d x_{1}^{4}-e x_{1}^{6}\right) & -b & -1
\end{array}\right] \\
\text { For } E(-0.99696,0,0,0), J \text { simplifies to }\left[\begin{array}{cccc}
0 & 1 & 0 & 0 \\
0 & 0 & 5 & 0 \\
0 & 0 & 0 & 1 \\
0 & 3.568 & -24 & -1
\end{array}\right] .
\end{gathered}
$$


The eigen values of the matrix $\mathrm{J}$ for $\mathrm{E}$ are:

$\mu_{1}=-0.853848+4.94767 i, \mu_{2}=-0.853848-4.94767 i, m_{3} 0.707697, \mu_{4}=0$

Here $\mu_{1}$ and $\mu_{2}$ are complex eigen values with negative real part, $\mu_{3}$ is a positive real eigen value and $\mu_{4}$ is zero eigen value which implies that $E$ is an unstable, non -hyperbolic equilibrium point.

\subsection{Lyapunov Exponents and Kaplan-Yorke Dimension}

Another way to determine the chaotic behavior of the system is to obtain the Lyapunov Exponents of the system. Lyapunov Exponents gives us a measure of the rate of convergence and divergence of infinitesimal close trajectories in the phase space. A positive lyapunov exponent confirms that the system under consideration is a chaotic one. Presence of more than one positive lyapunov exponent confirms hyper-chaotic behavior.

For parameter values $\mathrm{a}=5, \mathrm{~b}=24, \mathrm{c}=-0.05, \mathrm{~d}=2.55, \mathrm{e}=1.7, \mathrm{~g}=2.001$ and initial condition $(-0.1,0.2,-$ $0.1,0.1)$, the system shows the following lyapunov exponent dynamics depicted in Figure 3.

Figure 3. The Lyapunov Exponent Spectrum of System (1)

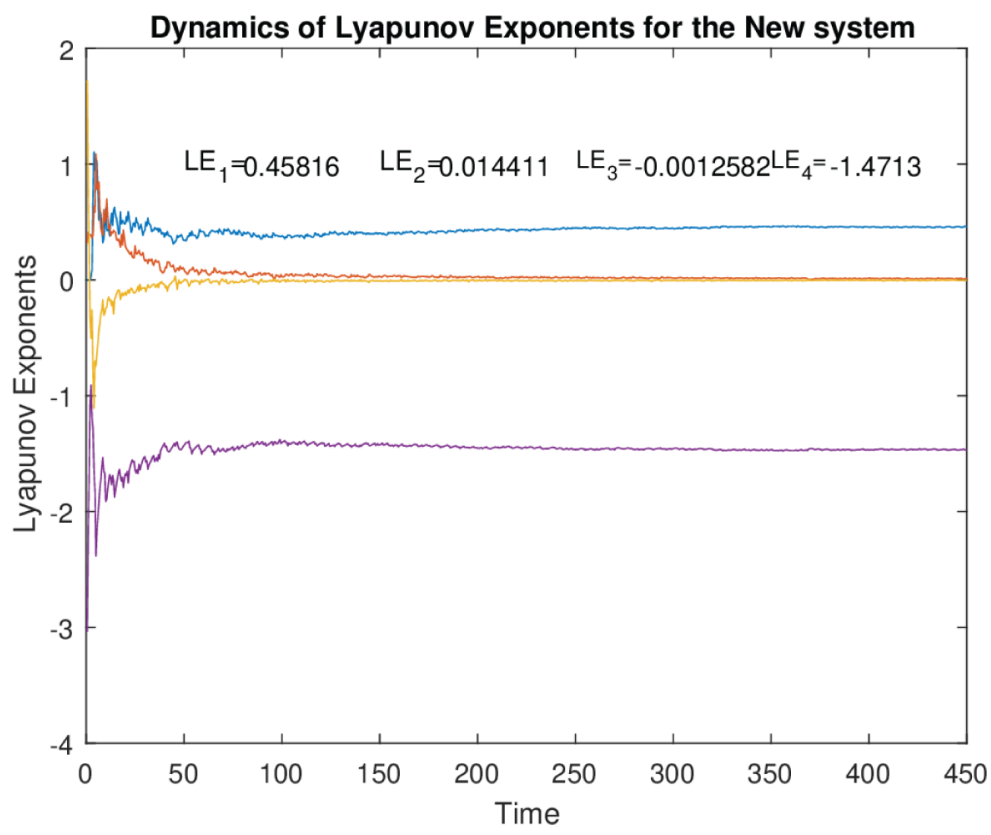

The numerical values of the lyapunov exponents as displayed in Figure 4 are:
L.E. $._{1}=.45816$
L.E. $._{2}=.014411$
L.E.3 $=-.0012582 » 0$
L.E. $4=-1.4713$ 
Figure 4. Poincare Section of system (1)

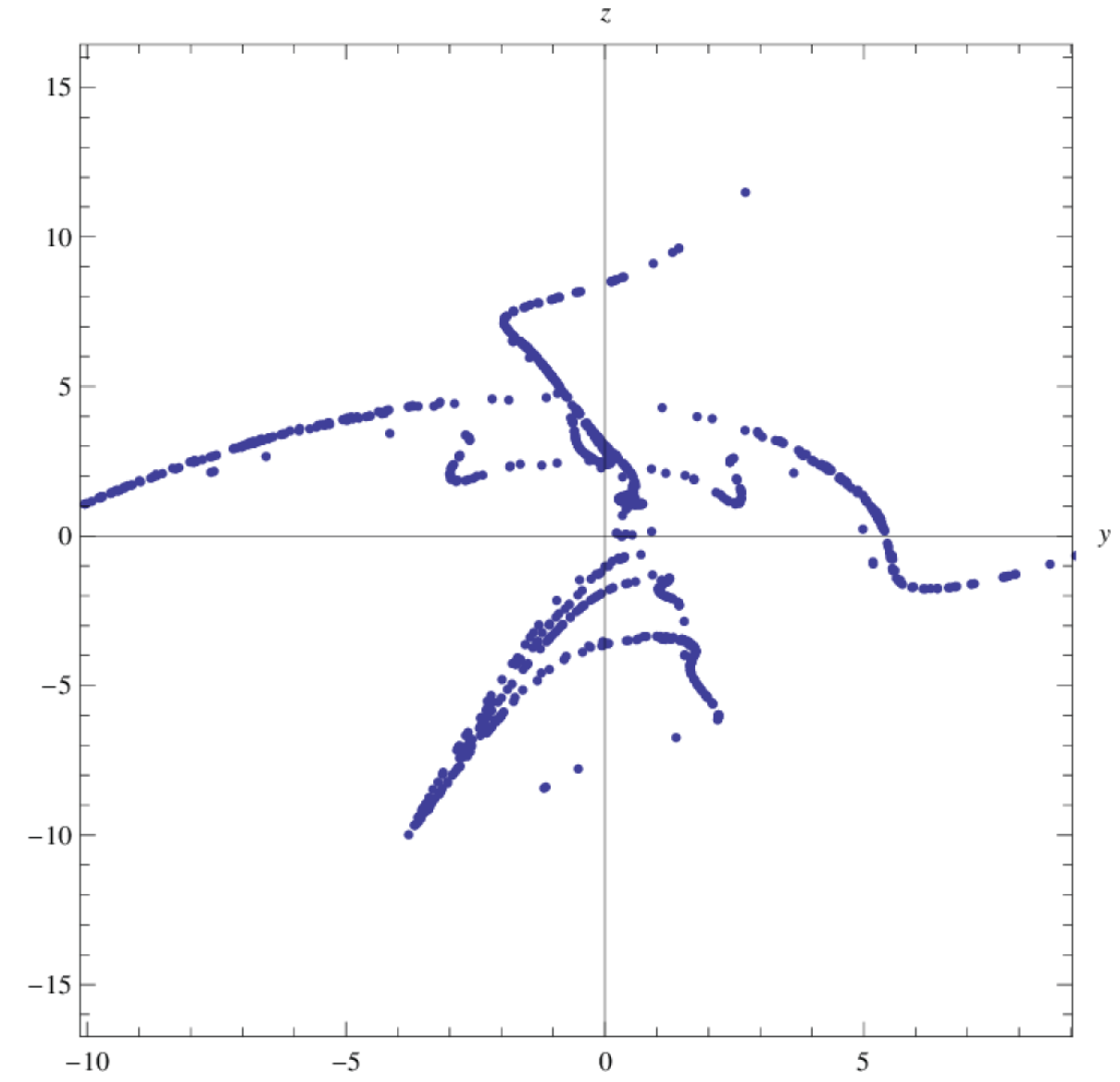

Also, the Kaplan-Yorke dimension is given by:

$D_{Y K}=m+\frac{\sum_{i=1}^{m} L \cdot E_{\bullet_{i}}}{\left|L \cdot E ._{r_{m+1}}\right|}$

where $m$ is the greatest number satisfying $\sum_{i=1}^{m} L . E ._{i} \geq 0$ and $\sum_{i=1}^{m+1} L . E ._{i}<0$.

Using the above Lyapunov Exponents values, we get the Kaplan-Yorke dimension as 3.320338 which is a non-integer value signifying highly complex behavior.

\subsection{Poincare Surface of Section and Bifurcation Analysis}

Poincare section is sketched to analyze the folding and bifurcating properties of chaotic system. The Poincare section of the system is shown in Figure 4. Here the denseness of the points depict existence of chaos in the system. 
Another method to analyze the changing dynamics of the system is to do the bifurcation analysis of the system by varying one parameter and keeping the other parameter values fixed. As the parameters of the chaotic system makes a significant influence on the dynamics and stability of the system,

Therefore it is necessary to observe the impact by varying the parameters on the system.

Here for the parameter values $a=5, b=24, c=-0.05, d=2.55, e=1.7, g=2.001$ and initial conditions $(-0.1,0.2,-0.1,0.1)$ the bifurcation diagrams for varying $a, b, c, d, e, f$ respectively are displayed in Figure 5. From Figures 5(a), 5(b), 5(c), 5(d), 5(e) and 5(f) it is clear that the system undergoes bifurcations and takes its route towards chaos over the range of parameters $a \in(1,5.5), b$ $\in(23,30), c \in(0,0.18), d \in(1,5), e \in(1.5,5)$ and $g \in(1,2.2)$.

Figure 5. Bifurcation diagram of system (1) for (a) $0 \leq a \leq 5.5$ (b) $23 \leq b \leq 30$ (c) $0 \leq c \leq 0.18$ (d) $1 \leq d \leq 5$ (e) $1.5 \leq e \leq 5$ (f) $1 \leq \mathrm{g} \leq \mathbf{2 . 2}$
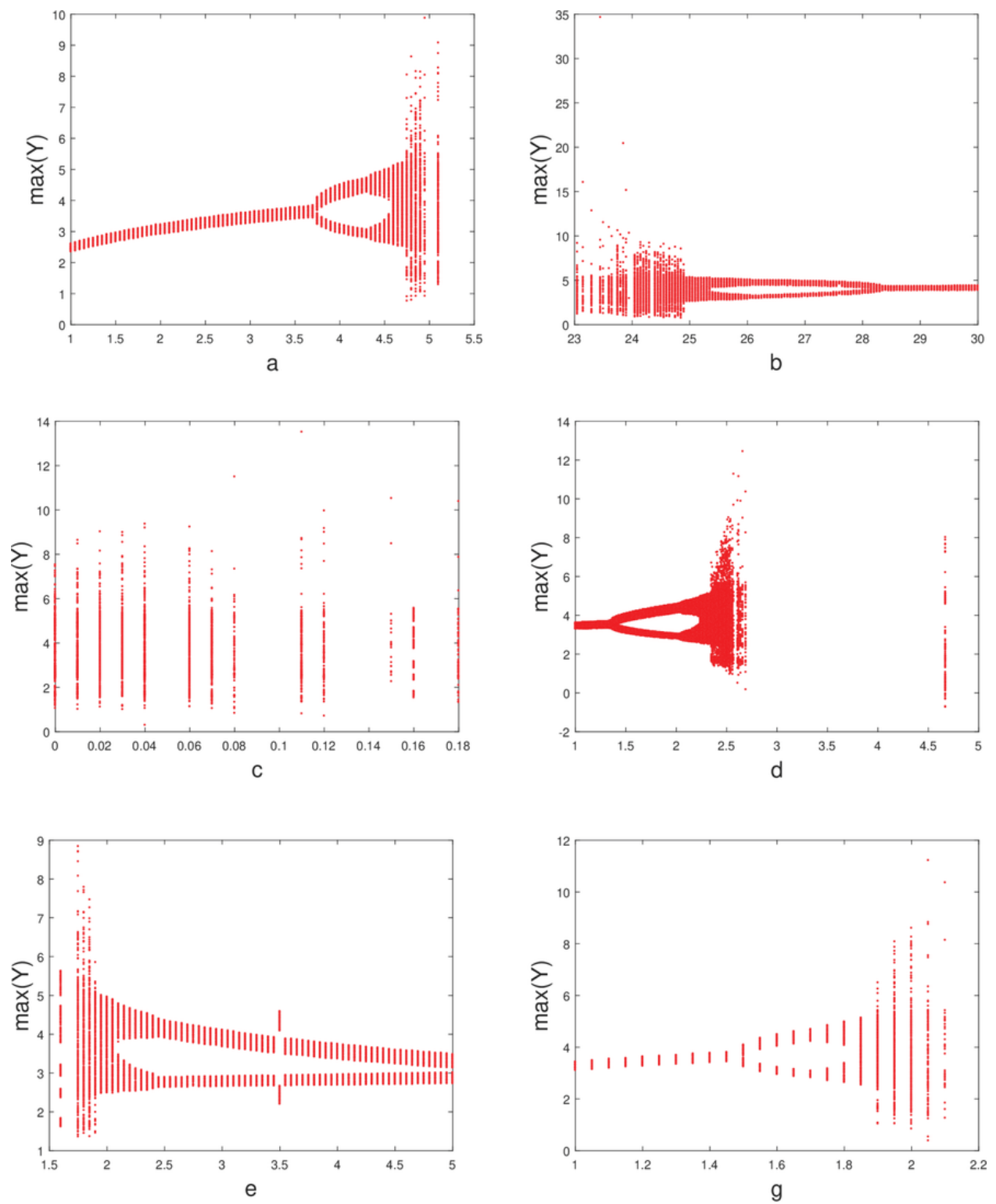


\section{SYNCHRONIZING THE IDENTICAL NEW 4-D HYPER-CHAOTIC SYSTEMS}

\subsection{Problem Formulation}

We first formulate the multi-switching compound difference synchronization scheme between the new 4-D hyper-chaotic systems. Taking three hyper-chaotic drive systems and one hyper-chaotic response system.

\subsubsection{Scaling Master System}

$\dot{V}=F(V)$

\subsubsection{Two Base Master Systems}

$\dot{W}=G(W)$

$\dot{X}=H(X)$

\subsubsection{Slave System}

$\dot{U}=E(U)+\bar{U}$

Here $V=\left(V_{1}, V_{2}, \ldots, V_{n}\right)^{T}, W=\left(W_{1}, W_{2}, . ., W_{n}\right)^{T}, X=\left(X_{1}, X_{2}, \ldots, X_{n}\right)^{T}, U=\left(U_{1}, U_{2}, \ldots U_{n}\right)^{T}$ are respectively the state variables of the system (5), (6), (7) and (8). F, G, H, E: $R^{n} \circledR R$ are continuous function and $\bar{U}=\mathbb{R}^{n} \times \mathbb{R}^{n} \times \mathbb{R}^{n} \times \mathbb{R}^{n} \rightarrow \mathbb{R}$ is the controller to be designed.

The multi-switching compound difference synchronization error is defined as:

$e_{i j k l}=A U-B V(C W-D X)$

where:

$A=\operatorname{diag}\left(\alpha_{1}, \alpha_{2}, \ldots a_{n}\right), B=\operatorname{diag}\left(\beta_{1}, \beta_{2}, \ldots, \beta_{n}\right), C=\operatorname{diag}\left(\gamma_{1}, \gamma_{2}, \ldots, \gamma_{n}\right), D=\operatorname{diag}\left(\delta_{1}, \delta_{2}, \ldots, \delta_{n}\right)$

and $A \neq 0$. Here $\mathrm{i}, \mathrm{j}, \mathrm{k}, 1$ represent the possible error switches which may assume any value between 1 to $n$.

For achieving the multi-switching compound difference synchronization between the response system and the compound of the drive systems we must have error tending to zero, i.e.:

$\lim _{t \rightarrow \infty}\left\|e_{i j k l}\right\|=\lim _{t \rightarrow \infty}\|A U-B V(C W-D X)\|=0$ 
Here we assume:

$U=\operatorname{diag}\left(U_{1}, U_{2}, \ldots, U_{n}\right), V=\operatorname{diag}\left(V_{1}, V_{2}, \ldots, V_{n}\right), W=\operatorname{diag}\left(W_{1}, W_{2}, \ldots, W_{n}\right), X=\operatorname{diag}\left(X_{1}, X_{2}, \ldots, X_{n}\right)$

\subsection{Synchronization Theory}

To achieve the desired synchronization among (5)-(8), the controllers are designed as:

$\bar{U}_{i}=\frac{\theta_{j k l}}{\alpha_{i}}-E_{i}-\frac{K_{i} e_{i j k l}}{\alpha_{i}}$

where:

$\theta_{j k l}=\beta_{i} F_{i}\left(\gamma_{k} W_{k}-\delta_{l} X_{l}\right)+\beta_{j} V_{j}\left(\gamma_{k} G_{k}-\delta_{l} h_{l}\right)$ for $j, k, l=1,2, \ldots, n$.

Theorem: Systems (5)-(7) will achieve the intended multi-switching compound synchronization with

(8) if the controllers are chosen as in equation (10).

Proof: The error as defined by (9) is given by:

$e_{i j k l}=\alpha_{i} U_{i}-\beta_{j} v_{j}\left(\gamma_{k} W_{k}-\delta_{l} X_{l}\right)$ for $i, j, k, l=1,2, \ldots ., n$

Hence the error dynamical system is given by:

$$
\begin{aligned}
& e_{i j k l}=\alpha_{i} \dot{U}_{i}-\beta_{j} \dot{V}_{j}\left(\gamma_{k} W_{k}-\delta_{l} X_{l}\right)-\beta_{j} V_{j}\left(\gamma_{k} \dot{W}_{k}-\delta_{l} \dot{X}_{l}\right) \\
& =\alpha_{i}\left(E_{i}-\dot{U}_{i}\right)-\beta_{j} F_{j}\left(\gamma_{k} W_{k}-\delta_{l} X_{l}\right)-\beta_{j} V_{j}\left(\gamma_{k} G_{k}-\delta_{l} H_{l}\right) \\
& =\alpha_{i}\left(E_{i}-\dot{U}_{i}\right)-\theta_{j k l}
\end{aligned}
$$

where:

$$
\theta_{j k l}=\beta_{j} F_{j}\left(\gamma_{k} W_{k}-\delta_{l} X_{l}\right)-\beta_{j} V_{j}\left(\gamma_{k} G_{k}-\delta_{l} H_{l}\right)
$$

Substituting the designed controller:

$$
\begin{aligned}
& e_{i j k l}=\alpha_{i}\left(E_{i}-E_{i}+\frac{\theta_{j k l}}{\alpha_{i}}-\frac{K_{i} e_{j k l}}{a_{i}}\right)-\theta_{j k l} \\
& =-K_{i} e_{i j k l}
\end{aligned}
$$

Define the Lyapunov function, a positive definite function as: 
$V(t)=\frac{1}{2} \sum_{i=1}^{n} e_{i j k l}^{2}$

where $\mathrm{e}_{\mathrm{ijkl}}$ denote the designed switches.

$$
\begin{aligned}
& \mathbf{P} \dot{V}(t)=\sum_{i=1}^{n} e_{i j k l} \dot{e}_{i j k l} \\
& =\sum e_{i j k l}\left(-K_{i} e_{i j k l}\right) \\
& =-\sum K_{i} e_{i j k l}^{2}
\end{aligned}
$$

We choose $\left(K_{1}, K_{2}, \ldots, K_{n}\right), K_{i}>0$ in such a way such that $\dot{V}(t)$ is negative definite. Therefore by Lyapunov Stability Theory we get $\lim _{t \rightarrow \infty}\left\|e_{i j k l}\right\|=0$, implying synchronization has been achieved.

\subsection{Illustration}

Scaling Master system: The scaling master system is given by:

$$
\begin{aligned}
& \dot{V}_{1}=V_{2} \\
& \dot{V}_{2}=a V_{3} \\
& \dot{V}_{3}=V_{4} \\
& \dot{V}_{4}=-b V_{3}-V_{4}+g V_{2}\left(1-c V_{1}^{2}+d V_{1}^{4}-e V_{1}^{6}\right)
\end{aligned}
$$

where $V=\left(V_{1}, V_{2}, V_{3}, V_{4}\right)$ are the state variables of the system and a, b, c, d, e, g are parameters. For $\mathrm{a}=5, \mathrm{~b}=24, \mathrm{c}=-0.05, \mathrm{~d}=2.55, \mathrm{e}=1.7, \mathrm{~g}=2.001$ and initial condition $(-0.1,0.2,-0.1,0.1)$ the system shows chaotic behavior.

\subsubsection{Base Master Systems}

The base master system-I and base master system-II are given respectively by:

$$
\begin{aligned}
& \dot{W}_{1}=W_{2} \\
& \dot{W}_{2}=a W_{3} \\
& \dot{W}_{3}=W_{4} \\
& \dot{W}_{4}=-b W_{3}-W_{4}+g W_{2}\left(1-c W_{1}^{2}+d W_{1}^{4}-e W_{1}^{6}\right)
\end{aligned}
$$


where $W=\left(W_{1}, W_{2}, W_{3}, W_{4}\right)$ are the state variables of the system and $\mathrm{a}, \mathrm{b}, \mathrm{c}, \mathrm{d}, \mathrm{e}, \mathrm{g}$ are parameters. For $\mathrm{a}=5, \mathrm{~b}=24, \mathrm{c}=-0.05, \mathrm{~d}=2.55, \mathrm{e}=1.7, \mathrm{~g}=2.001$ and initial condition $(0.2,0.2,0.2,0.2)$ the system shows chaotic behavior:

$$
\begin{aligned}
& \dot{X}_{1}=X_{2} \\
& \dot{X}_{2}=a X_{3} \\
& \dot{X}_{3}=X_{4} \\
& X_{4}=-b X_{3}-X_{4}+g X_{2}\left(1-c X_{1}^{2}+d X_{1}^{4}-e X_{1}^{6}\right)
\end{aligned}
$$

where $X=\left(X_{1}, X_{2}, X_{3}, X_{4}\right)$ are the state variables of the system and a, b, c, d, e, g are parameters. For $\mathrm{a}=5, \mathrm{~b}=24, \mathrm{c}=-0.05, \mathrm{~d}=2.55, \mathrm{e}=1.7, \mathrm{~g}=2.001$ and initial condition $(0.1,0.2,0.1,0.2)$ the system shows chaotic behavior.

\subsubsection{Slave System}

The slave system is described as:

$$
\begin{aligned}
& \dot{U}_{1}=U_{2}+\bar{U}_{1} \\
& \dot{U}_{2}=a U_{3}+\bar{U}_{2} \\
& \dot{U}_{3}=U_{4}+\bar{U}_{3} \\
& \dot{U}_{4}=-b U_{3}-U_{4}+g U_{2}\left(1-c U_{1}^{2}+d U_{1}^{4}-e U_{1}^{6}\right)+\bar{U}_{4}
\end{aligned}
$$

where $U=\left(U_{1}, U_{2}, U_{3}, U_{4}\right)$ are the state variables of the system and $\bar{U}_{1}=\left(\bar{U}_{1}, \bar{U}_{2}, \bar{U}_{3}, \bar{U}_{4}\right)$ are the controller to be designed. For $\mathrm{a}=5, \mathrm{~b}=24, \mathrm{c}=-0.05, \mathrm{~d}=2.55, \mathrm{e}=1.7, \mathrm{~g}=2.001$ and initial condition $(0.2,0.1,0.2,0.1)$ the system shows chaotic behavior.

Here, we assume $A=\operatorname{diag}\left(\alpha_{1}, \alpha_{2}, \ldots a_{n}\right), B=\operatorname{diag}\left(\beta_{1}, \beta_{2}, \ldots, \beta_{n}\right), C=\operatorname{diag}\left(\gamma_{1}, \gamma_{2}, \ldots, \gamma_{n}\right)$, $D=\operatorname{diag}\left(\delta_{1}, \delta_{2}, \ldots, \delta_{n}\right)$. The notations $\alpha_{i}, \beta_{i}, \gamma_{i}, \delta_{i}(i=1,2,3,4)$ representing the scaling factors are set for the convenience of discussion and may assume different or same values in applications.

We define the error $\left(e_{1}, e_{2}, e_{3}, e_{4}\right)$ as:

$$
\begin{aligned}
& e_{1}=e_{1234}=\alpha U_{1}-\beta_{2} V_{2}\left(\gamma_{3} W_{3}-\delta_{4} X_{4}\right) \\
& e_{2}=e_{2413}=\alpha_{2} U_{2}-\beta_{4} V_{4}\left(\gamma_{1} W_{1}-\delta_{3} X_{3}\right) \\
& e_{3}=e_{3214}=\alpha_{3} U_{3}-\beta_{2} V_{2}\left(\gamma_{1} W_{1}-\delta_{4} X_{4}\right)
\end{aligned}
$$


Figure 6. Trajectories of the Synchronized Master and Slave Systems

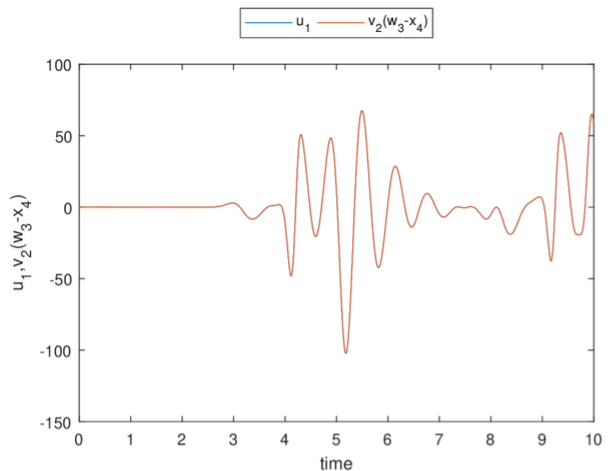

(a)

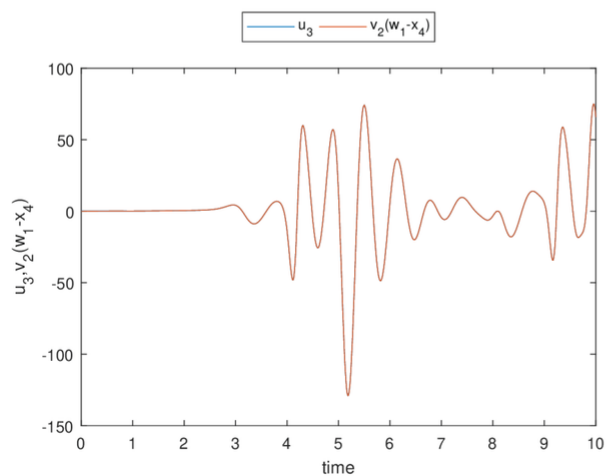

(c)

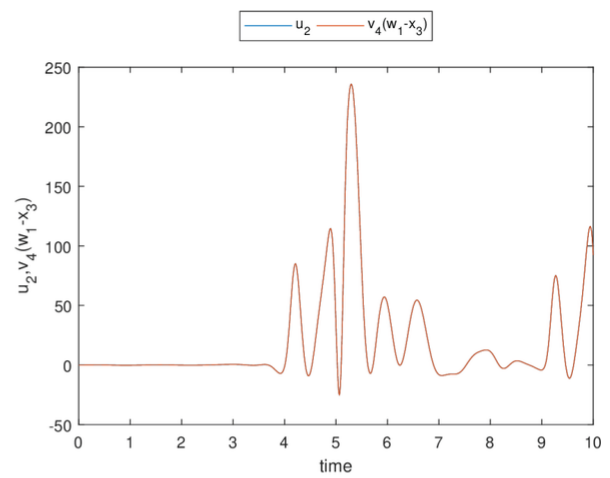

(b)

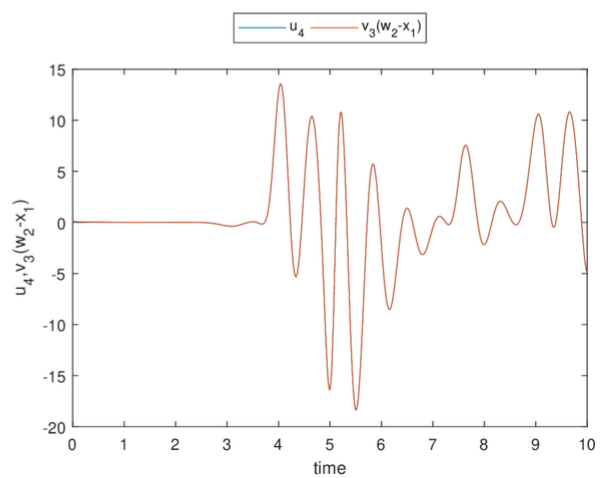

(d)

$$
e_{4}=e_{4321}=\alpha_{4} U_{4}-\beta_{3} V_{3}\left(\gamma_{2} W_{2}-\delta_{1} X_{1}\right)
$$

Therefore, the error dynamical system is obtained as:

$$
\begin{aligned}
& \dot{e}_{1}=\alpha_{1} \dot{U}_{1}-\beta_{2} \dot{V}_{2}\left(\gamma_{3} W_{3}-\delta_{4} X_{4}\right)-\beta_{1} V_{1}\left(\gamma_{1} \dot{W}_{1}-\delta_{1} \dot{X}_{1}\right) \\
& \dot{e}_{2}=\alpha_{2} \dot{U}_{2}-\beta_{4} \dot{V}_{4}\left(\gamma_{1} W_{1}-\delta_{3} X_{3}\right)-\beta_{4} V_{4}\left(\gamma_{1} \dot{W}_{1}-\delta_{3} \dot{X}_{3}\right) \\
& \dot{e}_{3}=\alpha_{3} \dot{U}_{3}-\beta_{2} \dot{V}_{2}\left(\gamma_{1} W_{1}-\delta_{4} X_{4}\right)-\beta_{2} V_{2}\left(\gamma_{1} \dot{W}_{1}-\delta_{4} \dot{X}_{4}\right) \\
& \dot{e}_{4}=\alpha_{4} \dot{U}_{4}-\beta_{3} \dot{V}_{3}\left(\gamma_{2} W_{2}-\delta_{1} X_{1}\right)-\beta_{3} V_{3}\left(\gamma_{2} \dot{W}_{2}-\delta_{1} \dot{X}_{1}\right)
\end{aligned}
$$

Substituting the value of the derivatives, error dynamical system simplifies to:

$$
\dot{e}_{1}=\alpha_{1}\left(U_{2}+\bar{U}_{1}\right)-\beta_{2}\left(\alpha V_{3}\right)\left(\gamma_{3} W_{3}-\delta_{4} X_{4}\right)-\beta_{1} V_{1}\left(\gamma_{1}\left(W_{2}\right)-\delta_{1}\left(X_{2}\right)\right)
$$




$$
\begin{gathered}
\dot{e}_{2}=\alpha_{2}\left(a U_{3}+\bar{U}_{2}\right)-\beta_{4}\left(-b V_{3}-V_{4}+g V_{2}\left(1-c V_{1}^{2}+d V_{1}^{4}-c V_{1}^{6}\right)\right)\left(\gamma_{1} W_{1}-\delta_{3} X_{3}\right) \\
-\beta_{4} V_{4}\left(\gamma_{1}\left(W_{2}\right)-\delta_{3}\left(X_{4}\right)\right) \\
\dot{e}_{3}=\alpha_{3}\left(U_{4}+\bar{U}_{3}\right)-\beta_{2}\left(a V_{3}\right)\left(\gamma_{1} W_{1}-\delta_{4} X_{4}\right)-\beta_{2} V_{2}\left(\gamma_{1}\left(W_{2}\right)-\delta_{4}\left(-b X_{3}-X_{4}\right.\right. \\
\left.+g X_{2}\left(1-c X_{1}^{2}+d X_{1}^{4}-e X_{1}^{6}\right)\right) \\
\dot{e}_{4}=\alpha_{4}\left(-b U_{3}-U_{4}+g U_{2}\left(1-c U_{1}^{2}+d U_{1}^{4}-e U_{1}^{6}\right)+\bar{U}_{4}\right)-\beta_{3}\left(V_{4}\right)\left(\gamma_{2} W_{2}-\delta_{1} X_{1}\right) \\
-\beta_{3} V_{3}\left(\gamma_{2}\left(a W_{3}\right)-\delta_{1}\left(X_{2}\right)\right)
\end{gathered}
$$

Figure 7. (a) (b), (c), (d). Error trajectories (e). The simultaneous error plot of the system.

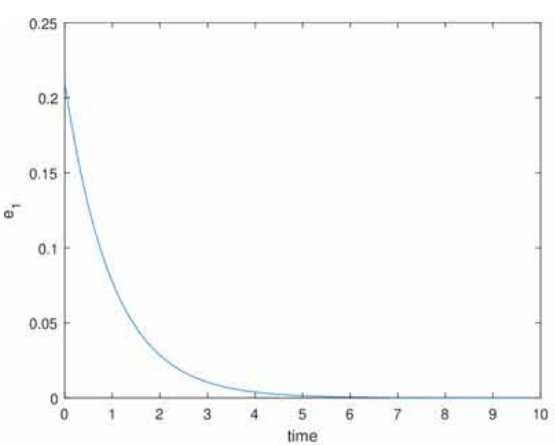

(a)

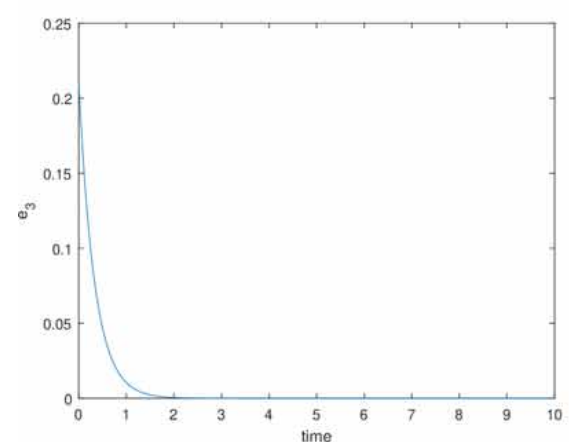

(c)

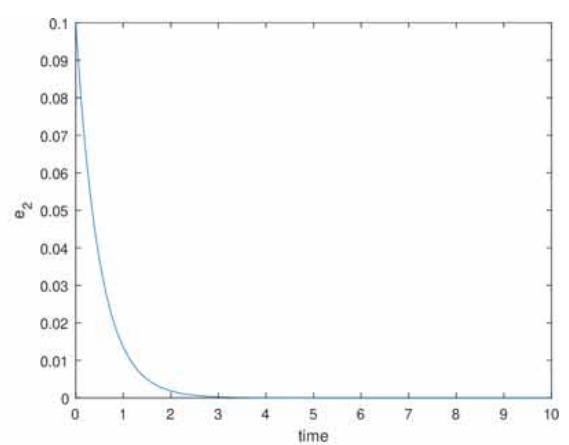

(b)

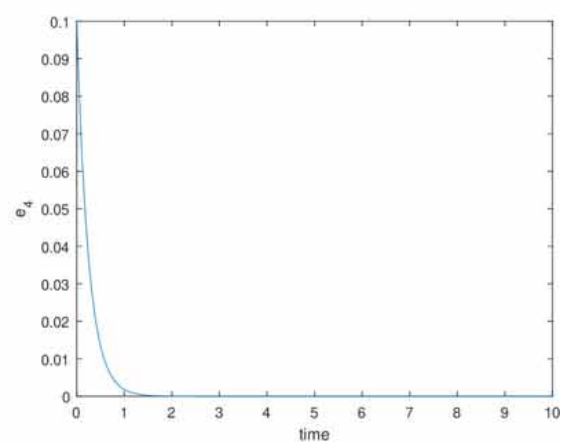

(d)

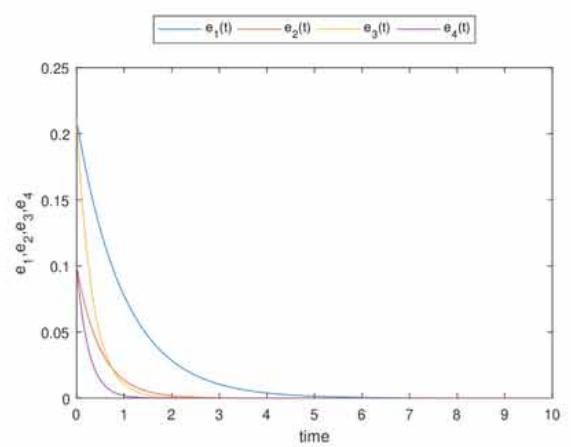

(e) 
The controllers are designed as:

$\bar{U}_{1}=-E_{1}(U)+\frac{\theta_{234}}{\alpha_{1}}-\frac{K_{1} e_{1}}{\alpha_{1}}$

where:

$\theta_{234}=\alpha_{2} F_{2}\left(\gamma_{3} W_{3}-\delta_{4} X_{4}\right)+\alpha_{2} V_{2}\left(\gamma_{3} G_{3}-\delta_{4} H_{4}\right)$

$\bar{U}_{2}=-E_{2}(U)+\frac{\theta_{413}}{\alpha_{2}}-\frac{K_{2} e_{2}}{\alpha_{2}}$

where:

$\theta_{413}=\alpha_{4} F_{4}\left(\gamma_{1} W_{1}-\delta_{3} X_{3}\right)+\alpha_{4} V_{4}\left(\gamma_{1} G_{1}-\delta_{3} H_{3}\right)$

$\bar{U}_{3}=-E_{3}(U)+\frac{\theta_{214}}{\alpha_{3}}-\frac{K_{3} e_{3}}{\alpha_{3}}$

where:

$\theta_{214}=\alpha_{2} F_{2}\left(\gamma_{1} W_{1}-\delta_{4} X_{4}\right)+\alpha_{2} V_{2}\left(\gamma_{1} G_{1}-\delta_{4} H_{4}\right)$

$\bar{U}_{4}=-E_{4}(U)+\frac{\theta_{321}}{\alpha_{4}}-\frac{K_{4} e_{4}}{\alpha_{4}}$

where:

$\theta_{321}=\alpha_{3} F_{3}\left(\gamma_{2} W_{2}-\delta_{1} X_{1}\right)+\alpha_{3} V_{3}\left(\gamma_{2} G_{2}-\delta_{1} H_{1}\right)$

Substituting (18) into (17), we get:

$\dot{e}_{1}=-K_{1} e_{1}$

$\dot{e}_{2}=-K_{2} e_{2}$

$\dot{e}_{3}=-K_{3} e_{3}$

$\dot{e}_{4}=-K_{4} e_{4}$

We now consider the Lyapunov function as: 


$$
\begin{aligned}
& V(t)=\frac{1}{2}\left(e_{1}^{2}+e_{2}^{2}+e_{3}^{2}+e_{r}^{2}\right) \\
& \dot{V}(t)=e_{1} \dot{e}_{1}+e_{2} \dot{e}_{2}+e_{3} \dot{e}_{3}+e_{4} \dot{e}_{4} \\
& =e_{1}\left(-K_{1} e_{1}\right)+e_{2}\left(-K_{2} e_{2}\right)+e_{3}\left(-K_{3} e_{3}\right)+e_{4}\left(-K_{4} e_{4}\right) \\
& =-K_{1} e_{1}^{2}-K_{2} e_{2}^{2}-K_{3} e_{3}^{2}-K_{4} e_{4}^{2}
\end{aligned}
$$

where $K_{1}, K_{2}, K_{3}, K_{4}$ are positive constants.

P $V(\dot{e}(t))$ is negative definite.

Therefore by the Stability Theory of Lyapunov we have that error vanishes with time, i.e. ei ! 0 for $i=1,2,3,4$. Hence the master systems (11)-(13) are now synchronized with the slave system (14).

\subsection{Results and Discussions}

Numerical Simulations have been done using MATLAB. We take here:

$$
\alpha_{1}=\alpha_{2}=\alpha_{3}=\alpha_{4}=\beta_{1}=\beta_{2}=\beta_{3}=\beta_{4}=\gamma_{1}=\gamma_{2}=\gamma_{3}=\gamma_{4}=\delta_{1}=\delta_{2}=\delta_{3}=\delta_{4}=1
$$

which means the slave system will completely synchronize with the compound of the multi-drive master systems. Also $K_{1}, K_{2}, K_{3}, K_{4}$ have been chosen to be $1,2,3,4$ respectively. The trajectories of the master systems (11)- (13) and slave system (14) are shown to get synchronized in Figure 6. Also the error plot of the system converges to zero and have been displayed in Figure 7 for the initial conditions $(0.21,0.1,0.21,0.1)$.

\section{PROPOSED APPLICATION OF SYNCHRONIZATION IN SECURE COMMUNICATION}

With the growing dependence on technology even for our smallest needs, it is important to ensure that it is hassle free and secure. The first and foremost concern in this regard is to protect the transmitted information from hackers as leakage of data could prove unimaginable loss.

To protect data leakage to hackers many methods are being developed. In the field of secure communication using the dynamics of the chaotic systems would be of great help owing to its sensitive dependence on initial conditions and parameter values.

The approach which has been developed here is based on the idea of simply burying the information signal containing the message within the chaotic signals obtained from a complex combination of the state variables of the novel chaotic system. The encoded message obtained using the chaotic dynamics makes the original message undistinguishable to the observer. Such a transmission increases the security of communicating the message as the encoded message resembling noise at the first sight is a complex function of message and chaotic variables. To decode the message at the receiving end the correct type of synchronization must be performed. Also, the parameter values and initial conditions of the chaotic systems must be exactly determined to extract the message. The property of chaotic systems to have entirely different dynamics at very very close initial conditions and parameter values adds to the security factor of such a transmission as it is impossible to guess values from among the in finite choices where the systems show chaotic behavior.

Here complex synchronization scheme has been used instead of the traditional synchronization method. Also, the presence of the non-standard hyper chaotic systems having two positive lyapunov exponents adds to the measure of security of the communication systems. 
We would now utilize the novel 4-D hyper-chaotic model in the field of secure communication using the multi-switching compound difference synchronization. An outline of the scheme has been shown in Figure 8.

Figure 8. Outline of the method in secure communication

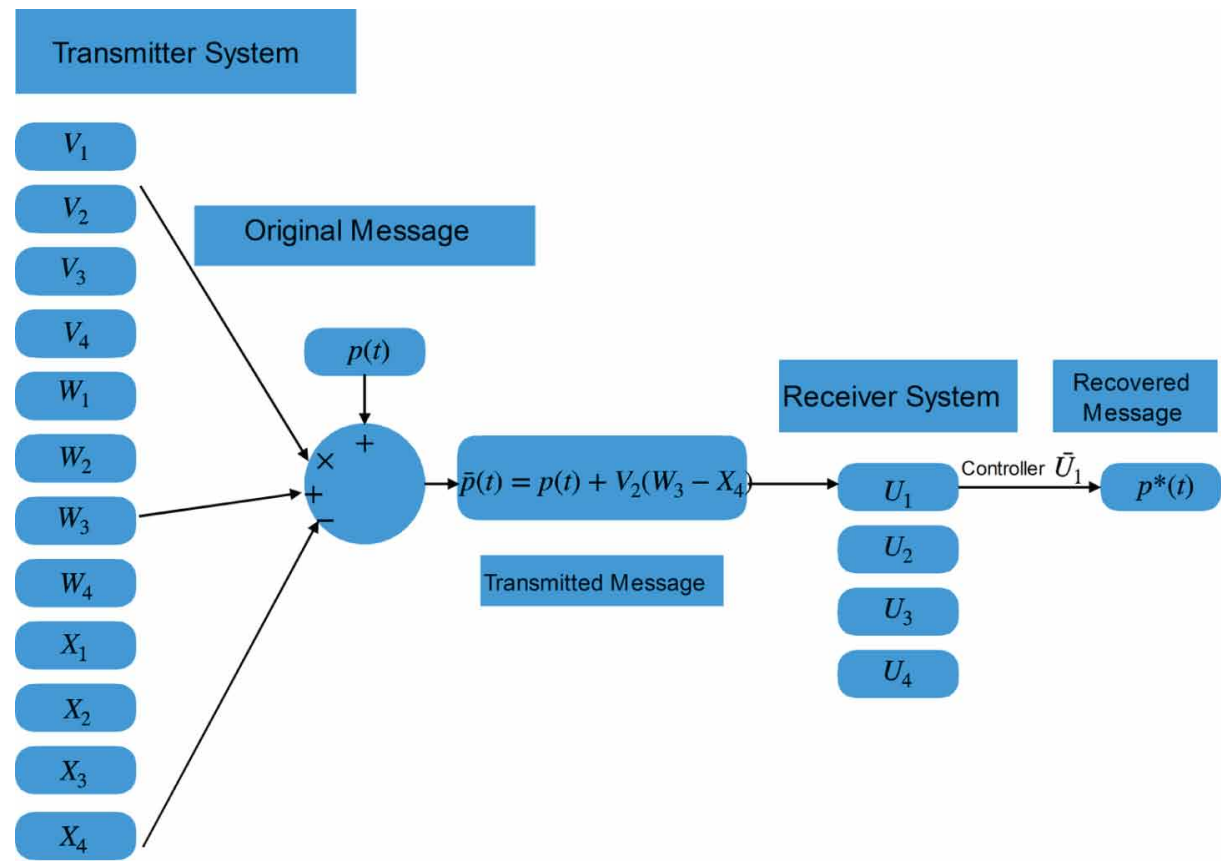

Let the message to be concealed be $p(t)$. We scramble it with disturbance forming a non-linear function composed of state variables of system at the transmitting end, say $p \overline{(t} t)=p(t)+$ disturbance.

Now we transmit $p \overline{(} t)$ to the receiving system where controllers are designed to synchronize with the scrambled disturbance formed by compound of master system and decode the transmitted message.

\subsection{Illustration}

We have applied the multi-switching compound difference synchronization on the above designed novel 4-D hyper-chaotic system. The parameter values and initial conditions are chosen as in Section 4.

Let the message to be transmitted be $p(t)=7 \sin (2 t)$.

We add it to the first equation of the compound of master systems and transmit i.e.:

$$
\bar{p}(t)=p(t)+V_{2}\left(W_{2}-X_{4}\right)
$$

On receiving the scrambled message, the controller $\bar{U}_{1}$ is added to variable $U_{1}$ to synchronize with $V_{2}\left(W_{3}-X_{4}\right)$ and decode the required message.

The original message to be sent is displayed in Figure 9 (a). The encoded/transmitted message is displayed in Figure 9 (b). The decoded/recovered message is displayed in Figure 9 (c). The error between the original message and recovered message is displayed in Figure 9 (d). 
Figure 9. (a) The original message (b) The transmitted message (c) The recovered message (d) Error Signal

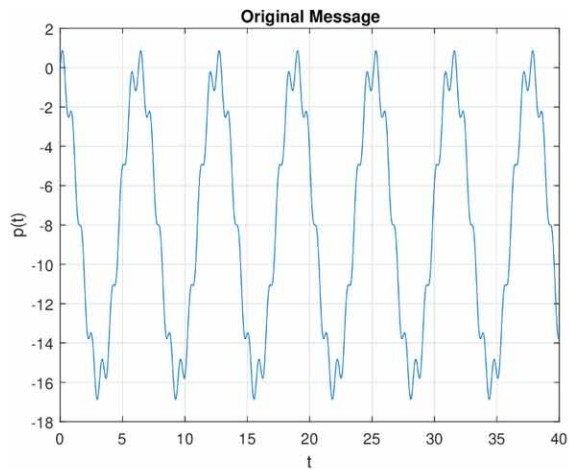

(a)

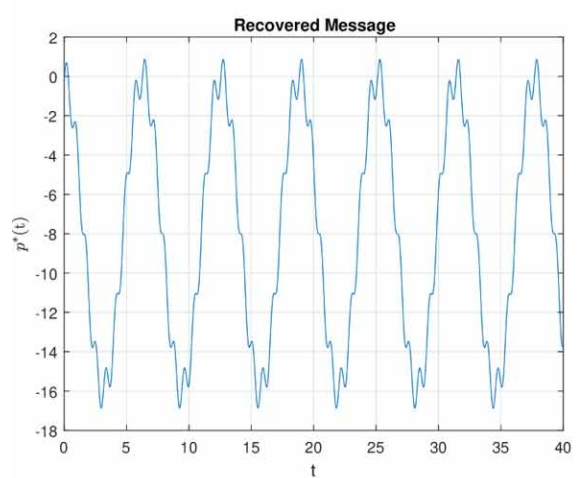

(c)

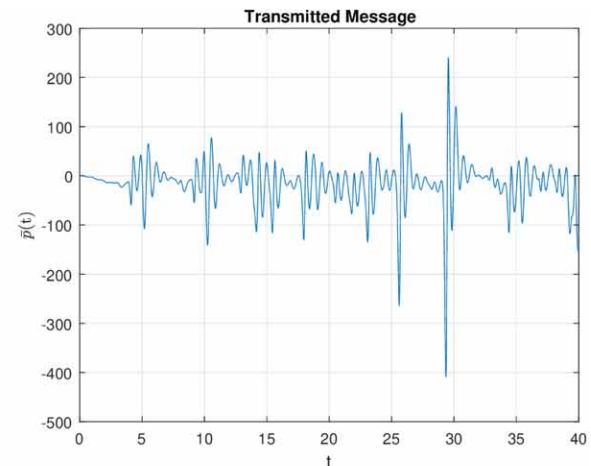

(b)

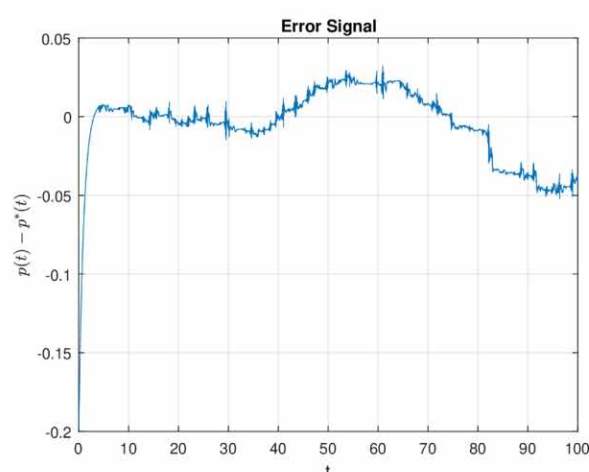

(d)

The above designed method would prove to be highly beneficial in the field of secure communication for the following reasons:

1. As the chaotic system is highly sensitive to initial conditions and parametric values, it is highly reliable in information exchange.

2. Performing synchronization using these non-standard chaotic systems has better chances of being unable to be guessed by the hackers as compared to the standard systems.

3. Here we have adopted the multi-switching compound difference synchronization scheme. This would promptly increase the security in data transmission as on one hand multi-switching would increase the possibilities in which variables have been synchronized, the compound difference would make the disturbances complex and hence difficult to decode the message.

\section{CONCLUSION}

In this manuscript a new 4-D hyper-chaotic system has been constructed and its dynamical analysis has been carried out by means of Lyapunov Exponent Spectrum, bifurcation diagram, phase portrait, time series, Poincare Surface of Section etc. Synchronization of these chaotic systems has also been carried out by using the novel technique "Multi-switching Compound Difference Synchronization". The application of the synchronization scheme developed has also been discussed in the field of secure communication. These studies would contribute to the understanding of the dynamical behavior in chaotic systems. 
Our future work will focus on the novel hyper-chaotic system's circuit realization, image encryption, exploring its hidden attractors, multi-stability, studies about fractional order chaotic systems with and without equilibrium points and other applications may include message encryption, random signal generation and other chaos based applications.

\section{ACKNOWLEDGMENT}

The first author is funded by the J.R.F. of C.S.I.R., India HRDG(CSIR)sanction letter no. 09/466(0189)/2017-EMR-I. 


\section{REFERENCES}

Balochian, S., \& Rajaee, N. (2018). Fractional-Order Optimal Control of Fractional-Order Linear Vibration Systems with Time Delay. International Journal of System Dynamics Applications, 7(3), 72-93.

Bouzaida, S., \& Sakly, A. (2018). Adaptive Neuro-Fuzzy Sliding Mode Controller. International Journal of System Dynamics Applications, 7(2), 34-54.

Broucke, M. (1987). One parameter bifurcation diagram for chua's circuit. IEEE Transactions on Circuits and Systems, 34(2), 208-209. doi:10.1109/TCS.1987.1086109

Chang, D., Li, Z., Wang, M., \& Zeng, Y. (2018). A novel digital programmable multi-scroll chaotic system and its application in fpga-based audio secure communication. AE $\dot{U}$. International Journal of Electronics and Communications, 88, 20-29. doi:10.1016/j.aeue.2018.03.007

Evans, D. J., Cohen, E., Searles, D. J., \& Bonetto, F. (2000). Note on the kaplan-yorke dimension and linear transport coefficients. Journal of Statistical Physics, 101(1-2), 17-34. doi:10.1023/A:1026449702528

Feki, M. (2003). An adaptive chaos synchronization scheme applied to secure communication. Chaos, Solitons, and Fractals, 18(1), 141-148. doi:10.1016/S0960-0779(02)00585-4

Geist, K., Parlitz, U., \& Lauterborn, W. (1990). Comparison of different methods for computing lyapunov exponents. Progress of Theoretical Physics, 83(5), 875-893. doi:10.1143/PTP.83.875

Ghabi, J., Rhif, A., \& Vaidyanathan, S. (2018). Discrete Time Sliding Mode Control Scheme for Nonlinear Systems With Bounded Uncertainties. International Journal of System Dynamics Applications, 7(2), 15-33.

Guo, W. (2011). Lag synchronization of complex networks via pinning control. Nonlinear Analysis Real World Applications, 12(5), 2579-2585. doi:10.1016/j.nonrwa.2011.03.007

Jafari, S., Pham, V.T., \& Kapitaniak, T. (2016). Multiscroll chaotic sea obtained from a simple 3d system without equilibrium. International Journal of Bifurcation and Chaos, 26(2).

Jafari, S., Sprott, J.C., \& Molaie, M. (2016). A simple chaotic flow with a plane of equilibria. International Journal of Bifurcation and Chaos, 26(6).

Jiang, C., Liu, S., \& Luo, C. (2014). A new fractional-order chaotic complex system and its antisynchronization. In Abstract and Applied Analysis (Vol. 2014). Hindawi.

Jiang, C., Zhang, F., \& Li, T. (2018). Synchronization and antisynchronization of n-coupled fractional-order complex chaotic systems with ring connection. Mathematical Methods in the Applied Sciences, 41(7), 2625-2638. doi:10.1002/mma.4765

Jimenez-Triana, A., Chen, G., \& Gauthier, A. (2015). A parameter-perturbation method for chaos control to stabilizing upos. IEEE Transactions on Circuits and Wystems. II, Express Briefs, 62(4), 407-411. doi:10.1109/ TCSII.2014.2387553

Karavaev, A., Kulminskiy, D., Ponomarenko, V., \& Prokhorov, M. (2015). An experimental communication scheme based on chaotic time-delay system with switched delay. International Journal of Bifurcation and Chaos, 25(10).

Khan, A., \& Bhat, M. A. (2016). Hybrid projective synchronization of fractional order chaotic systems with fractional order in the interval $(1,2)$. Non-linear Dyn Syst Theory, 16(4), 350-365.

Khan, A., \& Bhat, M. A. (2017). Multi-switching combination-combination synchronization of non-identical fractional-order chaotic systems. Mathematical Methods in the Applied Sciences, 40(15), 5654-5667. doi:10.1002/ mma.4416

Khan, A., \& Bhat, M. A. (2017). Multiswitching compound antisynchronization of four chaotic systems. Pramana, 89(6), 90. doi:10.1007/s12043-017-1488-7

Khan, A., Khattar, D., \& Prajapati, N. (2017). Reduced order multi switching hybrid synchronization of chaotic systems. Journal of Mathematical and Computational Science, 7(2), 414-429. 
Khan, A., Khattar, D., \& Prajapati, N. (2017). Adaptive multi switching combination synchronization of chaotic systems with un-known parameters. International Journal of Dynamics and Control, 6(2), 1-9.

Khan, A., \& Shikha, S. (2017). Increased and reduced order synchronisations between $5 \mathrm{~d}$ and $6 \mathrm{~d}$ hyperchaotic systems. Indian Journal of Industrial and Applied Mathematics, 8(1), 118-131. doi:10.5958/1945919X.2017.00010.X

Khan, A., \& Tyagi, A. (2018). Disturbance observer-based adaptive sliding mode hybrid projective synchronisation of identical fractional-order financial systems. Pramana, 90(5), 67. doi:10.1007/s12043-018-1555-8

Kousaka, T., Ueta, T., \& Kawakami, H. (1999). Bifurcation of switched non-linear dynamical systems. IEEE Transactions on Circuits and Systems. 2, Analog and Digital Signal Processing, 46(7), 878-885. doi: $10.1109 / 82.775383$

Li, C., Hu, W., Sprott, J. C., \& Wang, X. (2015). Multistability in symmetric chaotic systems. The European Physical Journal. Special Topics, 224(8), 1493-1506. doi:10.1140/epjst/e2015-02475-x

Li, C., \& Sprott, J.C. (2014). Multistability in the lorenz system: a broken butterfly. International Journal of Bifurcation and Chaos, 24(10).

Li, C., Sprott, J. C., \& Thio, W. (2015). Linearization of the lorenz system. Physics Letters. [Part A], 379(1011), 888-893. doi:10.1016/j.physleta.2015.01.003

Lu, J., Chen, G., Cheng, D., \& Celikovsky, S. (2002). Bridge the gap between the lorenz system and the chen system. International Journal of Bifurcation and Chaos in Applied Sciences and Engineering, 12(12), 2917-2926. doi:10.1142/S021812740200631X

Luo, A. C. J. (2009). A theory for synchronization of dynamical systems. Communications in Nonlinear Science and Numerical Simulation, 14(5), 1901-1951.

Mahmoud, G. M., \& Mahmoud, E. E. (2010). Phase and antiphase synchronization of two identical hyperchaotic complex nonlinear systems. Nonlinear Dynamics, 61(1-2), 141-152. doi:10.1007/s11071-009-9637-2

Mahmoud, G. M., \& Mahmoud, E. E. (2012). Lag synchronization of hyperchaotic complex nonlinear systems. Nonlinear Dynamics, 67(2), 1613-1622. doi:10.1007/s11071-011-0091-6

Othman, A A., Noorani, MSM., Al-Sawalha, M. M. (2017). Function Projective Dual Synchronization with Uncertain Parameters of Hyperchaotic Systems. International Journal of System Dynamics Applications, 6(4), $1-16$.

Park, J. H. (2005). Adaptive synchronization of hyperchaotic chen system with uncertain parameters. Chaos, Solitons, and Fractals, 26(3), 959-964. doi:10.1016/j.chaos.2005.02.002

Patra, P.K., Hoover, W.G., Hoover, C.G., \& Sprott, J.C. (2016). The equivalence of dissipation from gibbs entropy production with phase-volume loss in ergodic heat-conducting oscillators. International Journal of Bifurcation and Chaos, 26(5).

Pecora, L. M., \& Carroll, T. L. (1990). Synchronization in chaotic systems. Physical Review Letters, 64(8), 821-824. doi:10.1103/PhysRevLett.64.821 PMID:10042089

Pham, V.T., Jafari, S., Wang, X., \& Ma, J. (2016). A chaotic system with different shapes of equilibria. International Journal of Bifurcation and Chaos, 26(4).

Qin, W. X., \& Chen, G. (2007). On the boundedness of solutions of the chen system. Journal of Mathematical Analysis and Applications, 329(1), 445-451. doi:10.1016/j.jmaa.2006.06.091

Singh, A. K., Yadav, V. K., \& Das, S. (2016). Comparative study of synchronization methods of fractional order chaotic systems. Nonlinear Engineering, 5(3), 185-192. doi:10.1515/nleng-2016-0023

Skardal, P.S., Sevilla-Escoboza, R., Vera-Avila, V., \& Buldu, J.M. (2017). Optimal phase synchronization in networks of phase-coherent chaotic oscillators. Chaos: An Interdisciplinary Journal of Nonlinear Science, 27(1).

Torrieri, D.J. (1985). Principles of secure communication systems. Artech House, Inc. 
Vaidyanathan, S. (2014). Generalised projective synchronisation of novel 3-d chaotic systems with an exponential non-linearity via active and adaptive control. International Journal of Modelling. Identification and Control, 22(3), 207-217. doi:10.1504/IJMIC.2014.065339

Vaidyanathan, S., Volos, C. K., Kyprianidis, I., Stouboulos, I., \& Pham, V. T. (2015). Analysis, adaptive control and anti-synchronization of a six-term novel jerk chaotic system with two exponential nonlinearities and its circuit simulation. Journal of Engineering Science and Technology Review, 8(2).

Wang, X., Teng, L., \& Qin, X. (2012). A novel colour image encryption algorithm based on chaos. Signal Processing, 92(4), 1101-1108. doi:10.1016/j.sigpro.2011.10.023

Wei, Z. (2011). Dynamical behaviors of a chaotic system with no equilibria. Physics Letters. [Part A], 376(2), 102-108. doi:10.1016/j.physleta.2011.10.040

Wolf, A., Swift, J. B., Swinney, H. L., \& Vastano, J. A. (1985). Determining lyapunov exponents from a time series. Physica D. Nonlinear Phenomena, 16(3), 285-317. doi:10.1016/0167-2789(85)90011-9

Wu, Y., Noonan, J.P., Yang, G., \& Jin, H. (2012). Image encryption using the two-dimensional logistic chaotic map. Journal of Electronic Imaging, 21(1).

Wu, Z., Zhang, X., \& Zhong, X. (2019). Generalized chaos synchronization circuit simulation and asymmetric image encryption. IEEE Access: Practical Innovations, Open Solutions, 7, 37989-38008. doi:10.1109/ ACCESS.2019.2906770

$\mathrm{Yu}$, W. (1999). Passive equivalence of chaos in lorenz system. IEEE Transactions on Circuits and Systems. I, Fundamental Theory and Applications, 46(7), 876-878. doi:10.1109/81.774240

Zabihi, M., Kiranyaz, S., Rad, A. B., Katsaggelos, A. K., Gabbouj, M., \& Ince, T. (2016). Analysis of highdimensional phase space via poincare section for patient-specific seizure detection. IEEE Transactions on Neural Systems and Rehabilitation Engineering, 24(3), 386-398. doi:10.1109/TNSRE.2015.2505238 PMID:26701865 


\section{NOTATIONS}

- $\quad x=\left(x_{1}, x_{2}, x_{3}, x_{4}\right)^{T} \in R^{4}$ - State variables of the system

- $\quad a, b, c, d, e, g \in R$ - Parameters describing the system

- $\mathrm{V}(\mathrm{t})$ - Volume

- $\nabla S$ - Divergence of vector field $\mathrm{S}$

- E - Equilibrium points

- J - Jacobian matrix

- $\mu_{1}, \mu_{2}, \mu_{3}, \mu_{4}-$ Eigen values

- L.E. - Lyapunov exponent

- $\mathrm{D}_{\mathrm{Yk}}-$ Kaplan Yorke dimension

- $\mathrm{e}-$ Synchronization error

- $\mathrm{V}(\mathrm{t})$ - Lyapunov function

- $\alpha_{i}, \beta_{i}, \gamma_{i}, \delta_{i}-$ Scaling factors

- $\mathrm{K}_{\mathrm{i}}-$ Control gain matrix elements.

- $\mathrm{p}(\mathrm{t})$ - Original message to be sent

- $\bar{p}(t)$ - Transmitted message

- $p^{*}(t)$ - Recovered message

Pushali Trikha is research scholar at Department of Mathematics, Jamia Millia Islamia, New Delhi. Her area of interest is Chaos Theory and Synchronization.

Lone Seth Jahanzaib is research scholar at Department of Mathematics, Jamia Millia Islamia, New Delhi. His area of interest is Nonlinear dynamics. 